\title{
KNOWLEDGE AND PRACTISE OF DOCTORS AND NURSES ABOUT CHILDHOOD PNEUMOCCOCAL AND INFLUENZA VACCINES IN JORDAN
}

\author{
M.O. Ogundele ${ }^{1}$, H.F. Ayyash ${ }^{2}$, F.F. Ayyash ${ }^{3}$, R. Almardini ${ }^{3}$, C. Brett ${ }^{4}$
}

${ }^{I}$ Paediatrics, Alder Hey Royal Children's Foundation Hospital NHS Trust, Liverpool, ${ }^{2}$ Paediatrics, Doncaster Royal Infirmary, Doncaster, UK, ${ }^{3}$ Royal Medical Services, Queen Rania Abdulla Children's Hospital, Amman, Jordan, ${ }^{4}$ Audit and Research, Doncaster Royal Infirmary, Doncaster, UK

The WHO has issued recommendations for use of the conjugate pneumococcal vaccine and yearly influenza in children considered to be at high risk for both infections.

A survey of General Paediatricians and nurses in Jordan was undertaken in the winter of 2010, to assess their familiarity with the recommendations on pneumoccocal and influenza immunisation of high-risk children.

Method: Self-administered Questionnaires were sent to Paediatricians and nurses in 5 Community Child Health Centres (CCHC) and 10 District General hospitals (DGH).

Results: A total of 131 questionnaires (80\% of total) were returned from doctors $(60 \%)$ and nurses $(40 \%)$. One third of the respondents worked in DGH and the remaining in tertiary centres. $81 \%$ of the respondents were aware of the indications for the vaccines.

The most correctly identified high-risk children for both vaccines were cystic fibrosis (74\%), chronic lung disease $(71 \%)$ and HIV/AIDS (66\%) (Table 1). The least correctly identified conditions were Diabetes mellitus (34\%), Biliary atresia or chronic hepatitis (37\%) and chronic heart failure (48\%).

The preferred methods of advising patients included verbal information (53.4\%), the GP (44.2\%), and letters (20.5\%). $71.7 \%$ of respondents wanted the vaccines given in primary care, others preferred the tertiary centre $(31.3 \%)$ or the DGH $(22.9 \%)$. Monitoring adherence to vaccination was recommended by $84 \%$ through GP letters $(41.2 \%)$ or verbal information from carers $(54.2 \%)$.

Table 1 showing the distribution of correctly identified indication for the pneumococcal and

influenza vaccines.

\begin{tabular}{|c|c|c|c|c|}
\hline VACCINES & & PNEUMO & FLU & AVERAGE \\
\hline \multirow{5}{*}{$\begin{array}{l}\text { Children having a chronic } \\
\text { (ongoing) lung disease. }\end{array}$} & Cystic fibrosis & 74.8 & 73.3 & 74.05 \\
\hline & Chronic lung disease (CLD) & 74.8 & 67.9 & 71.35 \\
\hline & $\begin{array}{l}\text { Children previously admitted } \\
\text { with a lower respiratory tract } \\
\text { disease }\end{array}$ & NO (43.5) & 53.4 & 48.45 \\
\hline & $\begin{array}{l}\text { Asthmatics requiring regular } \\
\text { use of inhaled or systemic } \\
\text { steroids }\end{array}$ & 59.5 & 67.9 & 63.7 \\
\hline & $\begin{array}{l}\text { Children previously admitted } \\
\text { because of asthma }\end{array}$ & NO (39.7) & 67.9 & 53.8 \\
\hline $\begin{array}{l}\text { Children with respiratory } \\
\text { conditions caused by or } \\
\text { predisposing to aspiration. }\end{array}$ & $\begin{array}{l}\text { Neuromuscular disease (eg } \\
\text { cerebral palsy) }\end{array}$ & 51.9 & 64.9 & 58.4 \\
\hline \multirow{2}{*}{$\begin{array}{l}\text { Chronic heart disease e.g. } \\
\text { requiring regular medication } \\
\text { or follow-up }\end{array}$} & Congenital heart disease & 56.5 & 59.5 & 58 \\
\hline & Chronic heart failure. & 45 & 51.1 & 48.05 \\
\hline \multirow[t]{3}{*}{ Chronic kidney disease. } & Nephrotic syndrome & 69.5 & 58.8 & 64.15 \\
\hline & Chronic renal failure & 55 & 57.3 & 56.15 \\
\hline & Post renal transplant. & 64.9 & 54.2 & 59.55 \\
\hline \multirow[t]{2}{*}{ Chronic liver disease such as: } & $\begin{array}{l}\text { Biliary atresia, chronic } \\
\text { hepatitis }\end{array}$ & 35.9 & 38.2 & 37.05 \\
\hline & Cirrhosis. & 52.7 & 47.3 & 50 \\
\hline \multirow[t]{3}{*}{$\begin{array}{l}\text { Immunocompromised } \\
\text { patients }\end{array}$} & $\begin{array}{l}\text { Asplenia or severe splenic } \\
\text { dysfunction (eg homozygous } \\
\text { Sickle Cell or Coeliac disease). }\end{array}$ & 80.9 & 67.9 & 74.4 \\
\hline & HIV/AIDS & 71.8 & 61.1 & 66.45 \\
\hline & $\begin{array}{l}\text { On chemotherapy or steroid } \\
\text { treatment }\end{array}$ & 61.8 & 56.5 & 59.15 \\
\hline \multicolumn{2}{|l|}{ Diabetes mellitus } & 38.2 & No $(30.5)$ & 34.35 \\
\hline \multicolumn{2}{|l|}{ Children with cochlear implants } & 56.5 & NO (38.2) & 47.35 \\
\hline \multicolumn{2}{|l|}{ Patients with CSF shunts } & 63.4 & NO (27.5) & 45.45 \\
\hline \multicolumn{2}{|c|}{$\begin{array}{l}\text { Children under the age of } 5 \text { who have previously had invasive } \\
\text { pneumococcal disease }\end{array}$} & 72.5 & NO $(29.8)$ & 51.15 \\
\hline \multicolumn{2}{|c|}{ Average } & 58.44 & 53.66 & 56.05 \\
\hline
\end{tabular}

Conclusion: The low rate of correct identification of patients requiring pneumococcal and influenza vaccines among healthcare professionals in Jordan suggests a nation-wide programme of educational and increased awareness is urgently needed. 